\title{
Multiple moving person tracking by UWB sensors: the effect of mutual shielding persons and methods reducing its impacts
}

\author{
Dušan Kocur, Jana Fortes and Mária Švecová*
}

\begin{abstract}
Ultra-wideband (UWB) radars are sensors allowing to track people in critical environments and situations. The results reached by single UWB sensors for such applications have shown that they are able to detect and track a person very well in a single person scenario. However, in multiple moving person scenarios, the ability of a single UWB sensor to detect several persons is usually significantly reduced. This is caused by a mutual shielding among people. In this paper, we will deal with the mutual shielding effect and its impacts, as well as with the methods of improving multiple moving person tracking by UWB radars. Firstly, we will provide a comprehensive description of the mutual shielding effect. Then, based on its analyses, we will state three complementary approaches created by the authors of this paper to reduce its impacts. They include an enhancement of the low-level echo of the targets, radar antenna array positioning at a convenient height, UWB sensor network application and, finally, their mutual combinations. The properties of those approaches will be demonstrated by two experimental measurements aimed at through wall tracking of two and three people, respectively. The results obtained in the experiments will illustrate the mutual shielding effect and the potential of the methods we have proposed to reduce its impacts.
\end{abstract}

Keywords: Radar, Sensor, Sensor network, Shielding effect, Tracking, Weak signal enhancement

\section{Introduction}

The short-range localization capability is becoming one of the most attractive features of the ultra-wideband (UWB) radar systems. They allow detection and tracking of moving people with an advantage in critical environments (e.g. [1-3]) or under hindered conditions (e.g. humans located behind obstacles). It results from the fact that UWB radars operating in a frequency range of up to $5 \mathrm{GHz}$ are characterized by a good penetration of emitted electromagnetic waves through various materials [4], such as wood, brick, concrete, plastic, rock, ground, snow and etc. Such sensors can be portable, light-weight and small-sized and with a fine range resolution as well. These properties make the presented UWB sensor attractive for a wide range of security and safety applications, such as rescue [5] and law enforcement missions [6], behind-the-wall target

*Correspondence: Maria.Svecova@tuke.sk

Department of Electronics and Multimedia Communications, Faculty of Electrical Engineering and Informatics, Technical University of Košice, Letná 9, 04200 Košice, Slovak Republic detection and localization [7-14], surveillance and reconnaissance or sensing through smoke and dust, detection of people trapped after earthquakes or building collapses [15-18], monitoring of critical infrastructures (reservoirs, power plants, etc.) [2, 3], monitoring of elderly or handicapped people within ambient assisted living programs [19] and etc.

The problem of short-range detection and tracking of moving persons has been studied, e.g. in [12, 18, 20-24]. Its solution for single person scenarios can be considered successfully solved. However, the problem of multiple human tracking in a real complex environment has been less well addressed (e.g. [25]). The presence of multiple targets results in additional problems, such as ghost appearance, changing of target tracks or mutual shielding among people. Ghosts are defined as false targets originating for example in the localization phase when the measured bistatic ranges applied for the target position estimation do not belong to the same target [22]. To avoid ghosts, proper association of data received from all receiving channels needs to be done (e.g. $[22,26])$. The 
further level of data association is usually included into the complex multiple target tracking (MTT) system. It is responsible for the correct clustering of incoming observations and targets-to-tracks association during the whole observation time. The properly chosen MTT system also has to solve the problem of the changing number of targets and with the related maintenance of their tracks [27].

According to our opinion, the mutual shielding effect is the crucial problem appearing in multiple human tracking [28-31]. Our experiences with the short-range UWB radars dedicated to tracking of multiple moving persons have shown that very often only the target located near the radar antennas is visible. The other targets can also be detected but with less reliability than those located closer to the radar antennas. These frequently arising features due to the mutual shielding effect result in the essential reduction of the localization system performance efficiency.

In this paper, we would like to discuss methods enabling the increase of the probability of target detection in multiple person scenarios, and hence to improve the target tracking as well. Many useful articles reporting solutions for detection, localization and tracking of human beings can be found in the literature (e.g. [24, 32-37]). However, to our best knowledge, almost no paper (except e.g. [38]) addresses the problem of mutual shielding among people. Furthermore, there are only a few papers (e.g. [38]) which deal with multiple moving persons tracking that demonstrate the obtained results by using no simulations but experimental data. On that account, we will describe and illustrate the shielding effect and its impacts using appropriate experimental scenarios of multiple persons tracking (Section 2) and, subsequently, we will focus on the possible solutions to that problem (Sections 3-5). The presented approaches based on a weak signal enhancement, a suitable location of the UWB radar antenna array and application of the UWB sensor network have been described in the papers [23, 39-46] and summarized in our previous work [31]. In contrast to these papers, this contribution focuses on the deeper analyses of mutual shielding effect and detailed comparison of the performance of all proposed approaches and their combinations. Finally, the main conclusions and future work are summarized in Section 6.

\section{The effect of the mutual shielding of persons}

As it was outlined in Section 1, detection, localization and tracking of human beings by UWB sensors in emergency situations have attracted researchers and industry during the last decade. Experiments with handheld UWB sensors usually equipped with one transmitting and two receiving antennas have shown that the proposed solutions are capable of providing quite good localization and tracking of a single person. However, in so-called multiple moving person scenarios, the obtained results are not as good as in the single person scenarios. This finding can be explained as the impact of mutual shielding (shadowing) of persons located in the monitored area.

The effect of the mutual shielding of persons has been described for the first time in $[28,29]$. It has been shown in these papers that if a person is illuminated by electromagnetic waves emitted in the frequency band DC$5 \mathrm{GHz}$, then a shadow zone is created behind a person in the direction of the electromagnetic wave propagation. The shadow region can be defined as a region shielded from radar illumination by an object reflecting and/or absorbing electromagnetic waves emitted by the radar or reflected by a target $[47,48]$. In case of localization, a human body can also be considered such an object. Taking into account its permittivity, permeability and conductivity as well as the typical frequency band employed by a UWB sensor, a person reflects and absorbs the energy of electromagnetic waves emitted by the sensor transmitting antenna or reflected by another object or target [49]. The shadow region possesses approximately the shape of the arc of a circle with an infinite radius and with the center located in the position of the source of the electromagnetic wave emission (Figs. 1 and 2). Further simulations and measurements have shown that the attenuation due to the shielding effect can have values, e.g. up to $24 \mathrm{~dB}$ $[30,40]$. On the other hand, it should be stressed that the basic radar equation is still valid. Hence, the shielding effect results in an attenuation of the electromagnetic wave power received by the radar which is additional to that caused by the antenna parameters, distance between the antenna system and target, radar cross-sections and etc. The discussed high additional attenuation results in the decrease of the useful signal (target echo) to clutter and noise ratio and hence in a dramatic drop of the probability of the detection of a target located in the shadow region as well. In the next part of this section, we will try to explain and describe the shielding effect a bit more deeply than in our previous works [28-31]. These new findings will create a good basis for a suggestion of approaches improving the target detection, localization and tracking in the mentioned scenarios.

According to the motion states of the tracked persons, the shadow zone can take two forms. If a person creating the shadow zone is moving, then the shadow zone is space and time variables. Its shape and location with the additional attenuation levels are changing with the motion of particular persons. In the next, this kind of the shadow zone will be referred to as the dynamic one. On the other hand, the static shadow zone is created behind a motionless person. In that case, its shape and location and the additional attenuation levels are time-invariant. Illustration of the shadow zone creation is depicted in Figs. 1 and 2 . Here, the person localization by a two-node sensor 


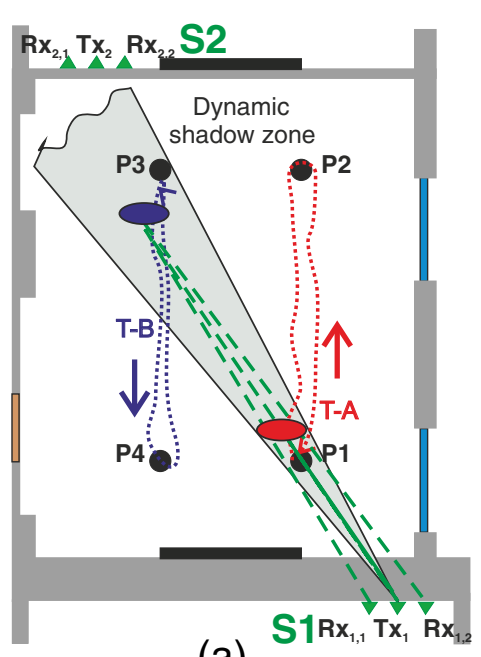

(a)

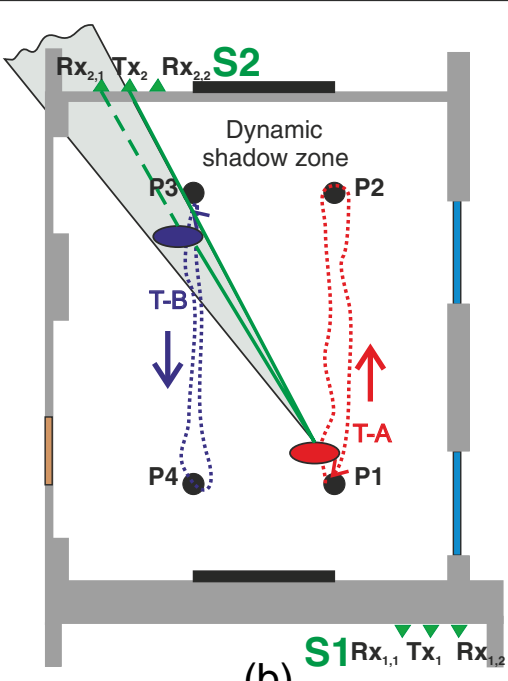

(b)

Fig. 1 The dynamic shadow zone creation model

network is assumed. The particular sensors (S1, S2) are represented by UWB radars equipped with one transmitting $\left(T x_{i}, i=1,2\right)$ and two receiving antennas $\left(R x_{i, j}\right.$ for $i=1,2, j=1,2)$.

For simplicity, it is considered here that the electromagnetic waves are transmitted by isotropic antennas and that the principles of ray optics can be used for the description of their propagation. The human bodies are substituted by the simple ellipses, and the presence of walls is ignored in the outlined schemes (Figs. 1 and 2). Although the ray optics-based approach is very simple, it is the good illustrative tool explaining the origin and basic properties of a shadow zone (e.g. its basic shape). This is the goal we follow in this section, and hence, we will not use any advance methods of electromagnetic field analyses here. A deeper description of the shadow zones focused on the distribution of the additional attenuation throughout a monitored area can be found in [30, 40, 50]. The results presented in these publications confirm that our simplified approach is really correct.

In Fig. 1, the dynamic shadow zone creation is illustrated. The target T-A is moving along the trajectory P1$\mathrm{P} 2-\mathrm{P} 1$. Figure 1a depicts the time instant when the target T-A is located in front of the transmitting antenna $\left(T x_{1}\right)$ of the sensor S1. It results in the occurrence of the shadow zone behind the target T-A. The zone is represented by

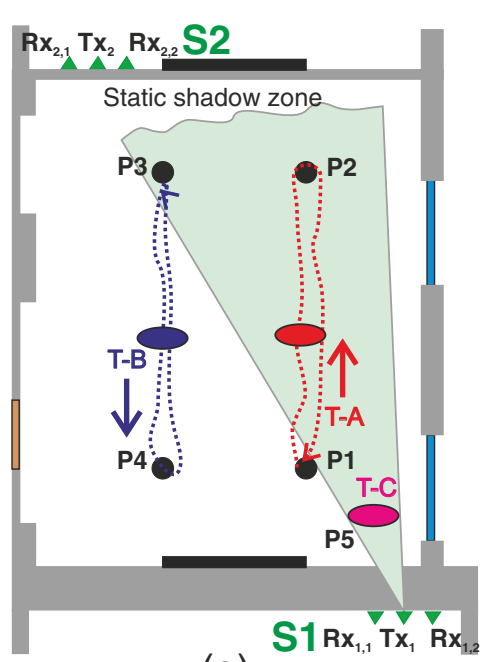

(a)

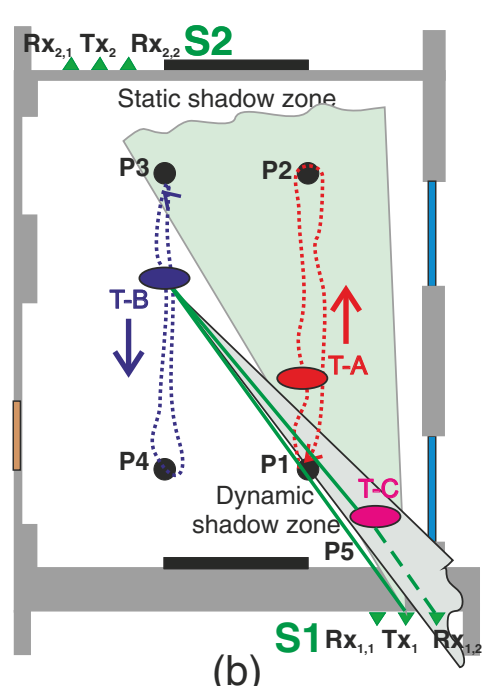

(b)

Fig. 2 The static shadow zone creation model 
the part of the grey arc of a circle located behind the T-A. Because the target T-B (moving along trajectory P3-P4P3) is at the same time located in this zone, he or she can reflect at best only strongly attenuated electromagnetic waves. And hence, it is difficult (at partial shielding) or impossible (at total shielding) to detect this target.

In Fig. 1b, the same scenario is analysed from the view of the sensor S2. Now, the target T-A can reflect the electromagnetic wave emitted by the transmitting antenna $T x_{2}$ of the S2 without any additional attenuation. However, between the target $\mathrm{T}-\mathrm{A}$ and the receiving antenna $R x_{2,1}$, the target T-B is located. And hence, T-B creates the shadow zone, too. This shadow zone is represented by the part of the grey arc of a circle located behind $\mathrm{T}-\mathrm{B}$. As the receiving antenna $R x_{2,1}$ is in regard of the $\mathrm{T}$-A located in this zone, the ability of S2 to detect the target $\mathrm{T}-\mathrm{A}$ by the receiving channel $R x_{2,1}$ is significantly reduced. Because the T-A and T-B are moving, the position and shape of the shadow regions sketched in Fig. 1a, b are changed with the movement of these targets. Therefore, these regions can be considered as dynamic shadow zones.

On the other hand, the formation of the static shadow zone is depicted in Fig. 2. In this case, the target T-C is standing directly in front of the antenna array of the sensor S1 including its transmitting antenna $\left(T x_{1}\right)$. Therefore, the shadow zone is created also by this target. The zone is represented by the part of the grey arc of a circle located behind T-C. Because the target T-C is standing, this shadow zone is the static one, i.e. it is invariant regarding its shape, location and additional attenuation. As it can be seen in Fig. 2a, the target T-A moving along the trajectory P1-P2-P1 is almost always in the outlined shadow region. And hence, it will be difficult or impossible to detect T-A by S1.
As is shown in Fig. 2a, b, the target T-B is moving along the reference positions P3-P4-P3. A part of this trajectory is located in the shadow region sketched in Fig. 2a. If the T-B is moving along this part of his or her trajectory, he or she is shadowed by the T-C. In this case, the shielding mechanism of the T-B is the same as shielding of the T-A by the T-C.

Another way of shielding can be observed if the T-B is moving outside of the shadow zone outlined in Fig. 2a. During this motion of T-B, the T-B can be illuminated by the transmitting antenna of S1 $\left(T x_{1}\right)$. However, the T-A can be located between T-B and receiving antenna $R x_{2,1}$. As the result, receiving antenna $R x_{2,1}$ of $\mathrm{S} 1$ is shielded by the $\mathrm{T}-\mathrm{A}$, and hence, the probability of the T-B detection will be decreased. And finally, if the T-B is moving outside of the shadow zone outlined in Fig. $2 \mathrm{~b}$, due to the mutual position of T-B, T-C and antenna array of S1, a dynamic shadow zone depicted in Fig. $2 \mathrm{~b}$ can be created as well. As the result, the probability of the T-B detection will be decreased once again.

The analyses of the scenarios according Figs. 1 and 2 have indicated that mutual shielding of persons could have a very severe impact on the probability of the target detection. In order to demonstrate the mutual shielding effect and its real impacts, the scenarios outlined above were realized at the school classroom according to the measurement scheme depicted in Fig. 3. In scenario 1, two persons (target T-A and target T-B) were walking at the same time against each other and then back through the reference positions P1-P2-P1 and P3-P4-P3 (Figs. 3a and 1). In scenario 2 , besides the two moving persons, a third person (target $\mathrm{T}-\mathrm{C}$ ) was standing during the whole measurement at the reference position P5 (Figs. 3a and 2).

The monitored area was scanned by two autonomous M-sequence UWB sensors [4], labelled S1 and S2, and

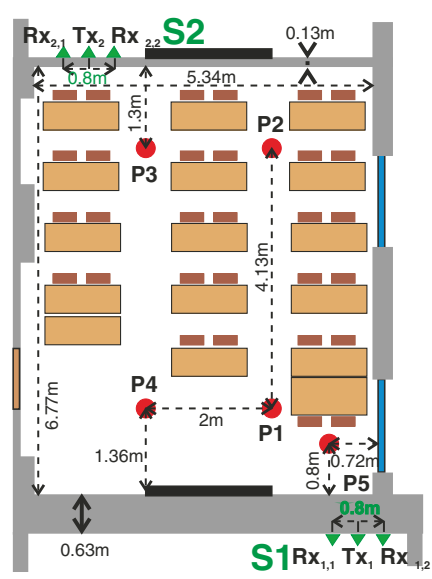

(a)

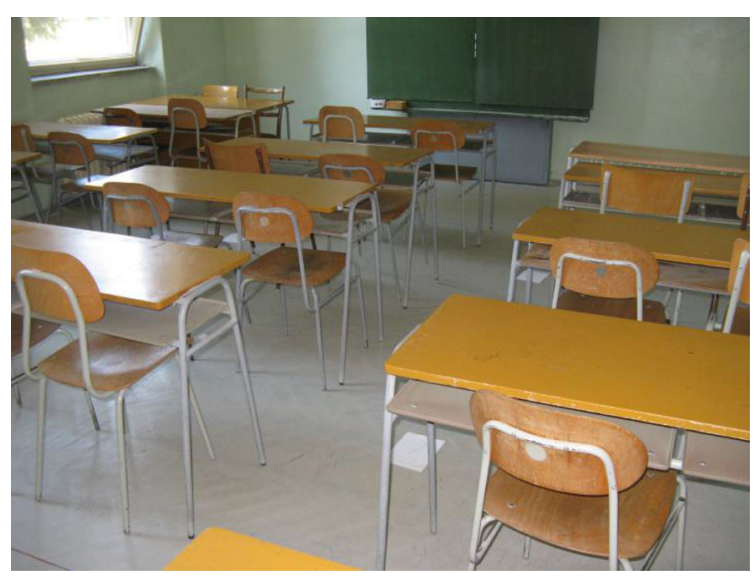

(b)

Fig. 3 Experimental scenario. a The measurement scheme. $\mathbf{b}$ The interior of the monitored area 
located behind concrete walls ( 0.13 and $0.63 \mathrm{~m}$ thick) in the adjacent classrooms (Figs. 3b and 4). Both sensors were equipped with three antennas [51] always placed in line with $T x_{i}$ in the middle between $R x_{i, 1}$ and $R x_{i, 2}$ for $i=1,2$. The distance between the adjacent antennas was set to $0.4 \mathrm{~m}$. The height of the antenna position was 1.35 or $1.85 \mathrm{~m}$ from the floor with no separation between the wall and the antennas. The number of antennas, their mutual positions and the distance among them correspond to a possible configuration of an antenna array of handheld UWB sensors.

Basic parameters of the sensors are summarized in Table 1. Both sensors have utilized cost-effective subsampling method for signal recording [4]. Moreover, in order to suppress random noise, a synchronous averaging of the impulse responses obtained by the subsampling approach was implemented in the radar devices. The combination of these approaches results in the measurement rates approximately 13.5 (S1) and 14.0 (S2) impulse responses per second, respectively. In our measurement, the obtained set of the impulse responses of the sensor S2 was off-line resampled and synchronized with the set of the impulse responses captured by the sensor S1. Then, the final joint measurement rate for S1 and S2 was 13.5 impulse responses per second. In our scenarios, both radar systems operated simultaneously over overlapping frequency bands. Because they emitted different $\mathrm{M}$-sequences at a different system clock frequency, their mutual interference was negligible.

The signals captured by both sensors were independently processed by the procedure of through wall tracking of multiple moving targets [52]. It consists of seven basic phases, such as background subtraction, target detection, time of arrival (TOA) estimation and TOA association, wall effect compensation, target localization and target tracking. For their implementation, the following methods were used: exponential averaging, constant
Table 1 Basic parameters of the sensors

\begin{tabular}{lll}
\hline & Sensor S1 & Sensor S2 \\
\hline System clock frequency & $4.5 \mathrm{GHz}$ & $13 \mathrm{GHz}$ \\
Operational bandwidth & $\mathrm{DC}-2.25 \mathrm{GHz}$ & $0.2-6.5 \mathrm{GHz}$ \\
M-sequence generator & linear, 9-stage & linear, 12-stage \\
Period of M-sequence & 511 chips & 4095 chips \\
Impulse responses & 511 samples/114 ns & 4095 samples / $315 \mathrm{~ns}$ \\
Maximum range & $17 \mathrm{~m}$ & $47 \mathrm{~m}$ \\
Range resolution & $3.33 \mathrm{~cm}$ & $1.15 \mathrm{~cm}$ \\
Output power & $1 \mathrm{~mW}$ & $10 \mathrm{~mW}$ \\
Antennas & $1 \mathrm{Tx}, 2 \mathrm{Rx} ;$ & $1 \mathrm{Tx}, 2 \mathrm{Rx} ;$ \\
& $\mathrm{Horn}$ antennas & Double-ridged \\
& & broadband antennas \\
\hline
\end{tabular}

false alarm rate (CFAR) detector [53, 54], trace connection method [26], trace correction of the 1st kind [55], direct method of localization [23, 56] and MTT system [23, 27] using linear Kalman filtering. The clarification of the particular phase importance and the description of methods of their implementations are beyond this paper. They can be found e.g. in $[23,57]$.

The results of radar signal processing for the analyzed scenarios are given in Figs. 5 and 6. In those figures, the detector outputs, the target localization results and the target track estimations are given. Now, we can discuss and analyze the obtained results.

In Fig. 5, the results obtained for scenario 1 by sensors S1 and S2 are depicted. The detector outputs consist of binary data, where the value "1" (white points) represents the reflection from the targets. The samples of the detector output corresponding to the same target create a so-called primary trace of a target. In Fig. 5a, b, almost the whole primary traces of the target T-A can be observed.

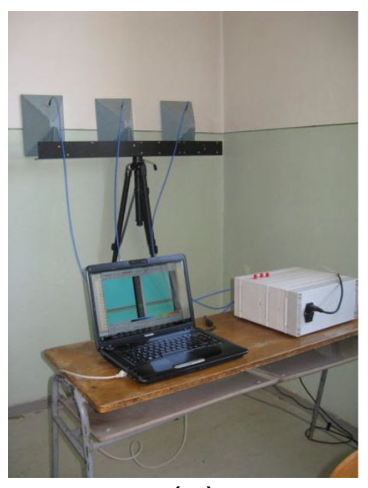

(a)

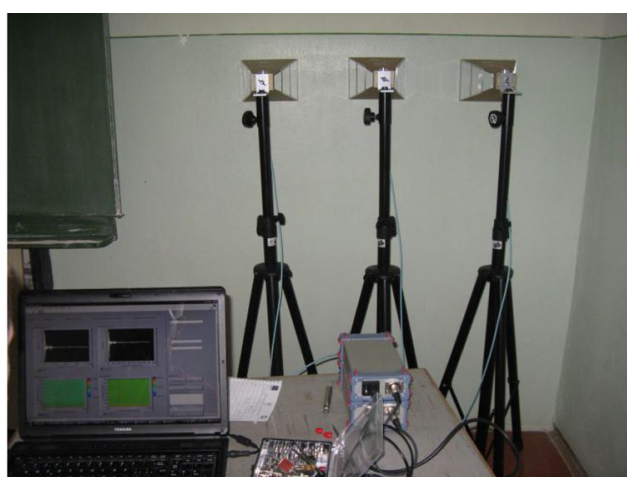

(b)

Fig. 4 The measurement devices and their locations. a Sensor S1. b Sensor S2 


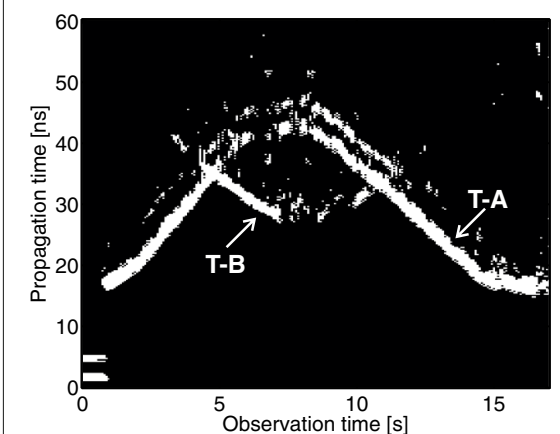

(a)

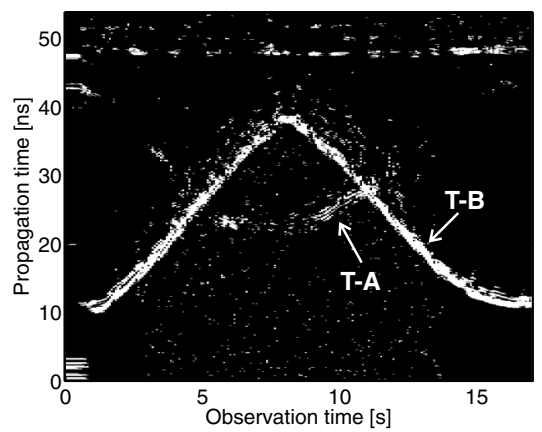

(d)

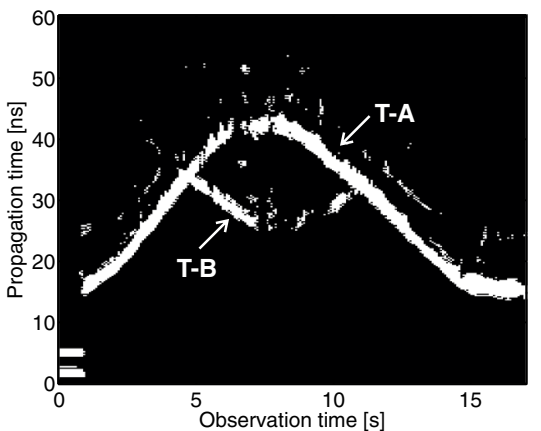

(b)

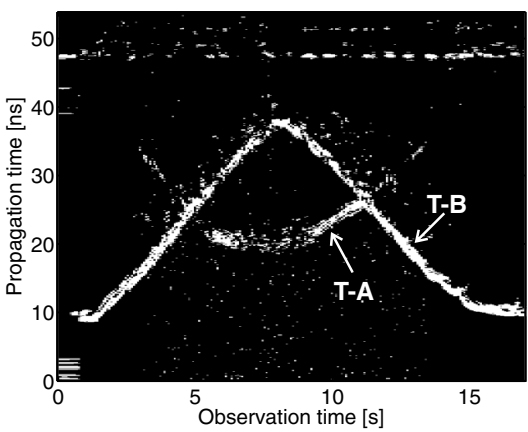

(e)

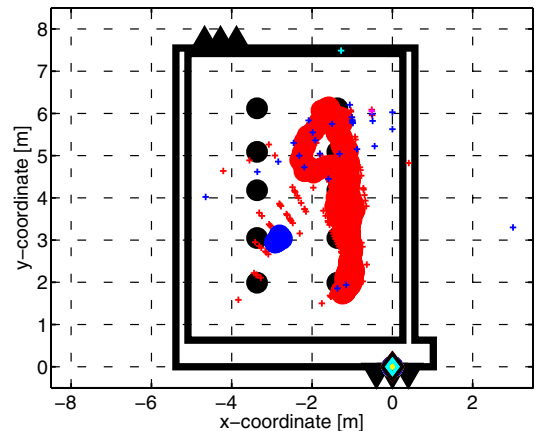

(c)

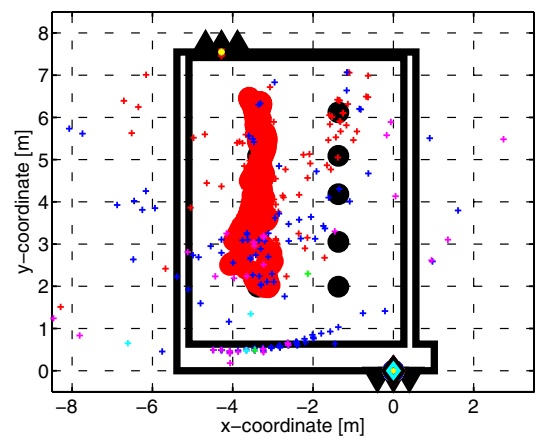

(f)

Fig. 5 Illustration of the impact of dynamic shielding (scenario 1). Detection, localization (plus sign symbols) and tracking results obtained by the sensor S1: a detector output for $R x_{1,1}, \mathbf{b}$ detector output for $R x_{1,2}$ and $\mathbf{c}$ final target tracks. Detection, localization (plus sign symbols) and tracking results obtained by the sensor S2: d detector output for $R x_{2,1}$, e detector output for $R x_{2,2}$ and $\mathbf{f}$ final target tracks

In contrast, the target $\mathrm{T}-\mathrm{B}$ was detected by $\mathrm{S} 1$ only during a short observation interval when T-B was moving in the vicinity of the position P4. Figure $5 \mathrm{c}$ shows the outputs of the localization (plus sign symbols) and tracking phase (circles). In accordance with detection results, the T-A track is estimated completely with a localization imprecision only in those segments of the target tracks where channels $R x_{1,1}$ and $R x_{1,2}$ did not provide data confirming the presence of the target T-A. The track of the target T-B emerges only for a while (blue circles in Fig. $5 \mathrm{~d}$ ) which occurs due to the poor detection of that target. The second row of Fig. 5 depicts the analogical outputs for signals captured by the sensor S2. Now, the presence of T-B is fully detected. At the same time, T-A is shielded most of the observation time (Fig. $5 \mathrm{~d}$, e). As channels $R x_{2,1}$ and $R x_{2,2}$ provide insufficient detection of T-A even in the not shadowed area, the estimated target locations were isolated and failed to confirm the existence of a new track. Hence, Fig. $5 \mathrm{f}$ contains the track of the target T-B only.

Figure 6 depicts the results obtained in scenario 2. In Fig. $6 \mathrm{a}, \mathrm{b}$ the detected target $\mathrm{T}-\mathrm{C}$ is represented by the white points creating a wide strip in the bottom parts of the detector outputs of sensor S1. The differences between Figs. 5a, b and 6a, b illustrate the consequences of the static shadow zone generated by T-C. As can be observed in Fig. 6a, b, the primary traces of T-A and T-B are markedly weaker than that due to T-C. It causes the poor results of the localization and tracking of T-A and T$\mathrm{B}$ depicted in Fig. 6c. Here, the position of standing person $\mathrm{T}-\mathrm{C}$ is estimated correctly. The T-A track (blue track) is estimated only partially in the farther positions from the shielding person $\mathrm{T}-\mathrm{C}$, where the shadow zone is getting weaker. Finally, the T-B track has not started at all. From the view of sensor S2, T-C is located far behind the trajectories of T-A and T-B. Therefore, its presence has no influence on the detection of T-A and T-B. On the other side, T-C is markedly shielded during the motion of T-A and $\mathrm{T}-\mathrm{B}$, and therefore, its position is not estimated by $\mathrm{S} 2$ (Fig. 6f). In view of these findings, we can interpret the results of the detection, localization and tracking for scenario 2, sensor S2 and targets T-A and T-B in the same way as the similar results for scenario 1 , sensor $\mathrm{S} 2$ and targets $\mathrm{T}-\mathrm{A}$ and $\mathrm{T}-\mathrm{B}$.

We can summarize the results of our analyses of the mutual shielding effect and the experimental results of scenarios 1 and 2 as follows:

- In case of multiple moving person localization, a dynamic and/or static shadow region is created. If a 


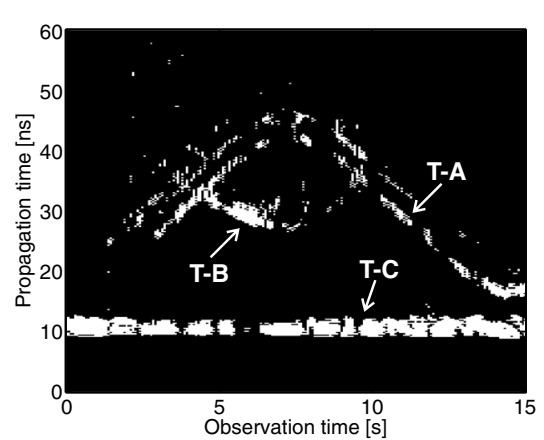

(a)

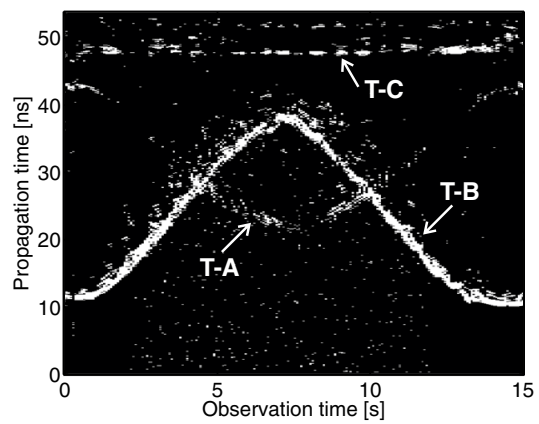

(d)

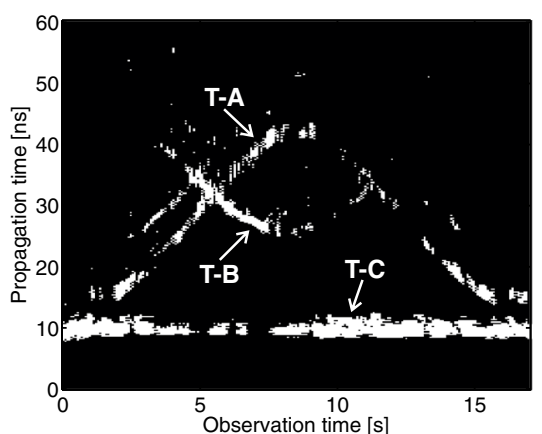

(b)

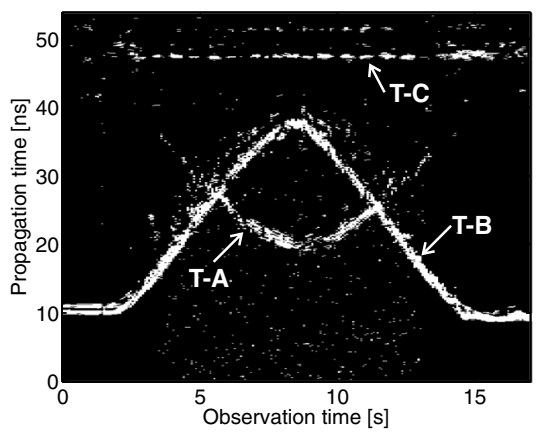

(e)

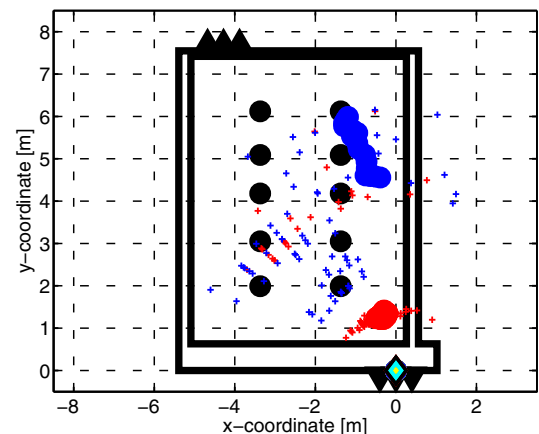

(c)

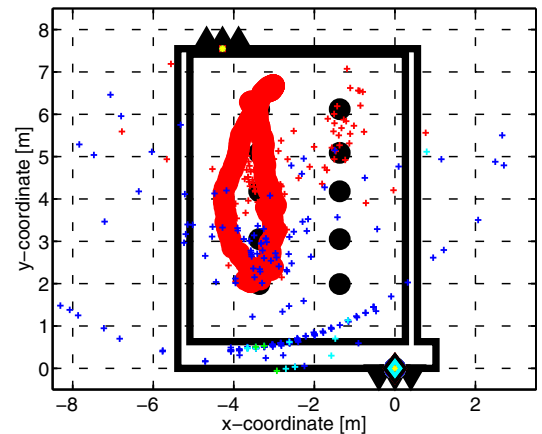

(f)

Fig. 6 Illustration of the impact of static shielding (scenario 2). Detection, localization (plus sign symbols) and tracking results obtained by the sensor S1: a detector output for $R x_{1,1}, \mathbf{b}$ detector output for $R x_{1,2}$ and $\mathbf{c}$ final target tracks. Detection, localization (plus sign symbols) and tracking results obtained by the sensor S2: $\mathbf{d}$ detector output for $R x_{2,1}$, e detector output for $R x_{2,2}$ and $\mathbf{f}$ final target tracks

person is situated in the shadow region, the probability of his/her detection is decreased.

- The shadow region is created by the person(s) located before a transmitting antenna and/or the person(s) located between a receiving antenna and another person (target).

- The additional attenuation due to the shielding effect is changing within the shadow region. The additional attenuation results in a dramatic drop of the detection probability of the target located in the shadow region. It results in:

- TOA estimation probability decrease (e.g. TOAs for one or for both receiving channel is not possible to estimate).

- Target localization probability decrease (e.g. one or both TOAs are missing, or any acceptable association of TOAs which would correspond to the same target cannot be reached).

- Due to the target detection probability drop, targets are sometimes localized and sometimes not. Depending on the length of the continuous time interval within which the target is localized, the MTT will create and maintain the target tracks. If the target is localized rarely, then the target tracks will not be created or will disappear.

Taking into account those facts, multiple target tracking improvement can be achieved in the following ways:

- Increasing a target echo-to-clutter and noise ratio. To solve this problem, low-level target echo enhancement by the advanced normalization method can be used $[23,41]$.

- Reducing the level of the additional attenuation due to mutual shielding. This objective can be achieved for example by a suitable location of the radar antenna system.

- Direct extension of the continuous time intervals in which the targets are localized. It can be achieved for example by an UWB sensor network application $[39,42]$.

Then, new concepts of multiple person localization and tracking with an improved performance can be obtained as the combination of the mentioned UWB radar signal processing procedure with the approaches listed in the previous paragraph. Block diagrams of these concepts are shown in Figs. 7 and 8. In the next 


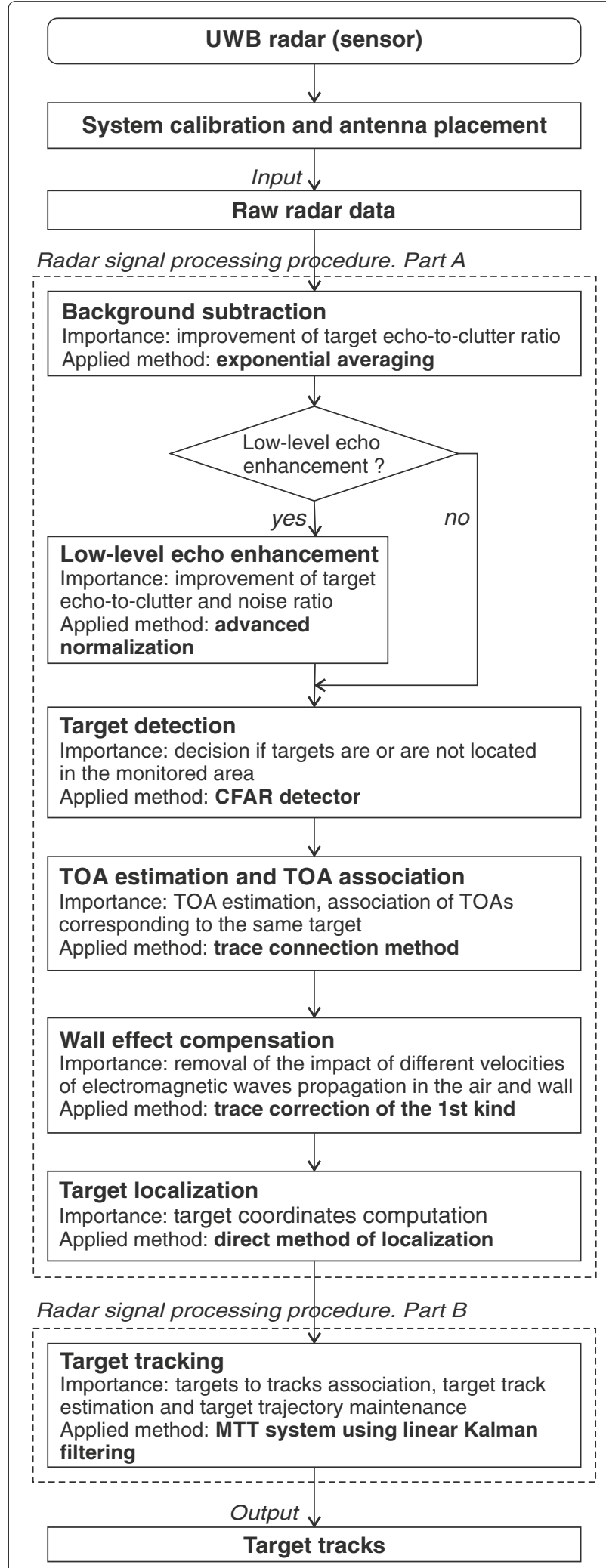

Fig. 7 Block diagram of radar signal processing procedure for a single UWB radar

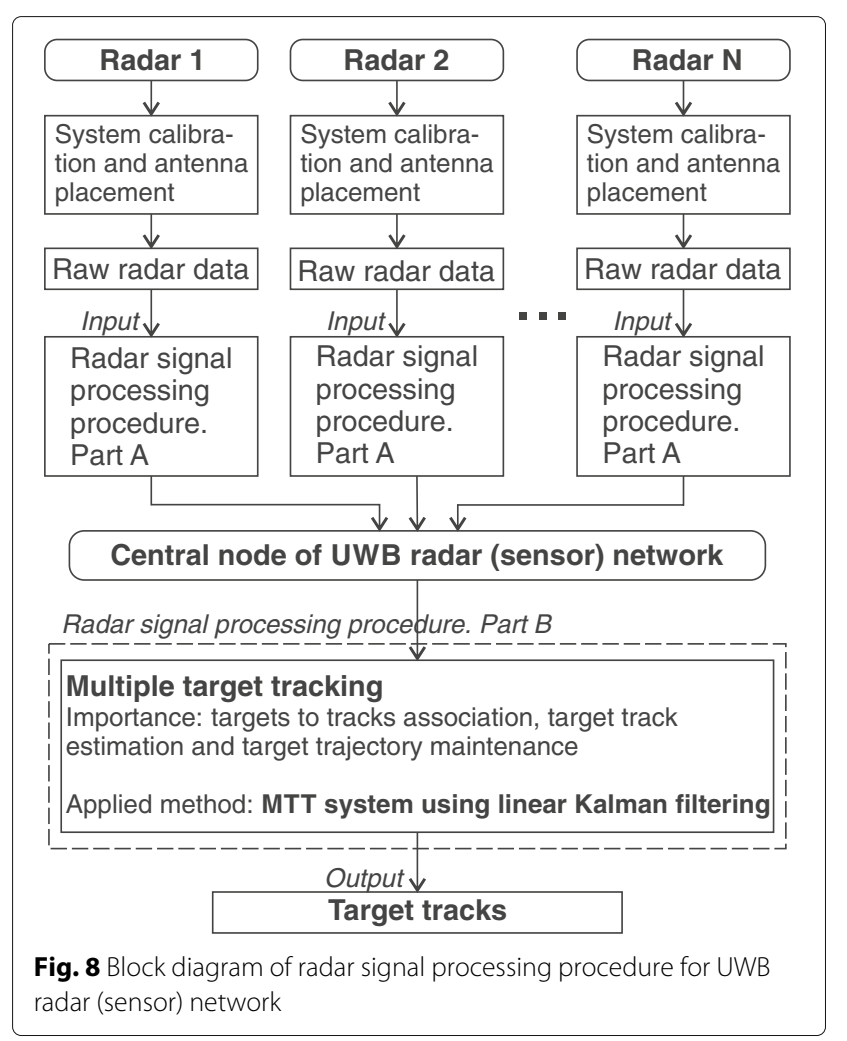

sections, we will describe the proposed concepts a little more detail.

\section{Low-level echo enhancement}

As was already outlined in the previous section, the target located close to the sensor antennas is able to produce strong reflections, whereas another target located far from the antenna system reflects a weaker echo. This effect, strengthened by mutual shielding between people, can result in the target signatures disappearing from the sensor response. In such cases, target detection can be improved by the application of advanced signal processing methods aimed at a weak signal enhancement. For example, a nonlinear enhancing of weak signals using optimization theory, neural network-based filters, weighing of the impulse responses and automatic gain control method can be used for that purpose $[41,58]$.

According to our findings, the method referred to as advanced normalization, introduced in [41], provides the most effective enhancement of the weak non-stationary signal components corresponding to a target echo. This simple method is based on successive searching for maxima of the impulse response with the subtracted background and a successive normalization of signal segments defined by these maxima. This approach allows to enhance the particular segments of the impulse response in a different way. A detailed description of the advanced 
normalization method is beyond this paper. A reader having an interest in this topic can find more examples in [23]. Taking into account the promising potential of the advanced normalization outlined in [41], we have decided to use this method for the low-level echo enhancement for the scenarios analyzed in this paper. The results confirming relevance of this choice will be shown in the next sections.

\section{Suitable location of the radar antenna array}

The application of the advanced normalization method results in increased probability of the detection of mutually shielded persons [31]. However, it holds only in scenarios where the additional attenuation is not very high (so-called partial shielding). Therefore, a great effort has been put into searching for the further methods of reducing the total shielding effect impact (i.e. cases where the additional attenuation is very high). Here, a very interesting and the same time simple solution is provided by the radar antenna array location at an appropriate relative altitude (i.e. the vertical distance of the antennas from a plane in which the target is localized) $[40,50]$. A simple and intuitive analysis of that idea has shown that the relative altitude of the radar antenna location has a significant impact on receiving of a line-of-sight component of the backscatters (particularly in the case of a single target scenario). On the other hand, the antenna location influences the level and distribution of the additional attenuation throughout a monitored area as well (mostly in the case of multiple target scenario when shadow regions are created). Taking into account these facts, the impact of the relative altitude of the radar antenna location on the person detectability has been studied in detail in [40] and [50]. As follows from these works, the location of the radar antennas at the altitude of the human head or a bit higher (approximately 180-210 cm) could increase the probability of the detection of the target located in the shadow zone.

The outlined approach (i.e. the appropriate location of the radar antennas) can be combined with the methods of low-level echo enhancement. The success of this idea has been confirmed in [31]. It has been shown in that paper that the mentioned combined approach has allowed detection and tracking even of a totally shielded person.

The impact of the weak signal enhancement application and radar antenna location on the target detection in scenario 2 and for sensors S1 and S2 are illustrated in Figs. 9 and 10, respectively. The particular detector outputs have been obtained under the following conditions:

- No signal enhancement or advanced normalization has been applied to weak signal enhancement.

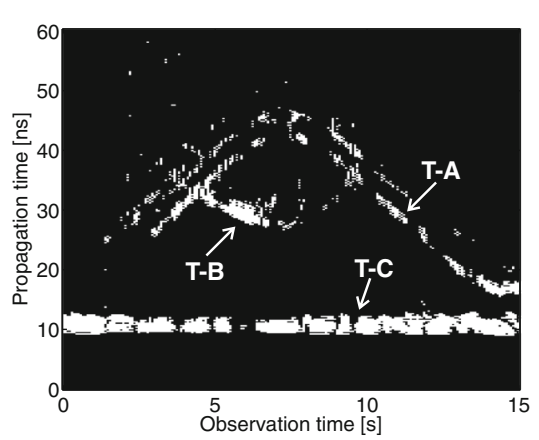

(a)

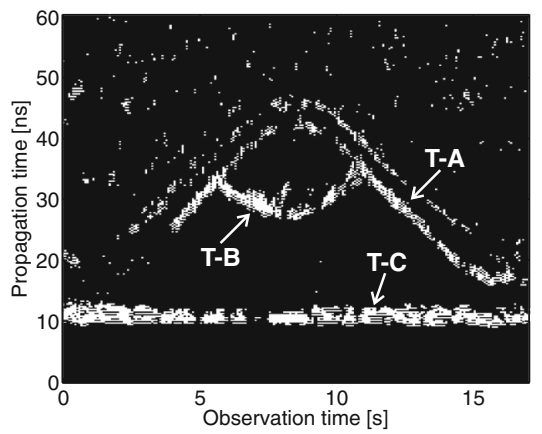

(b)

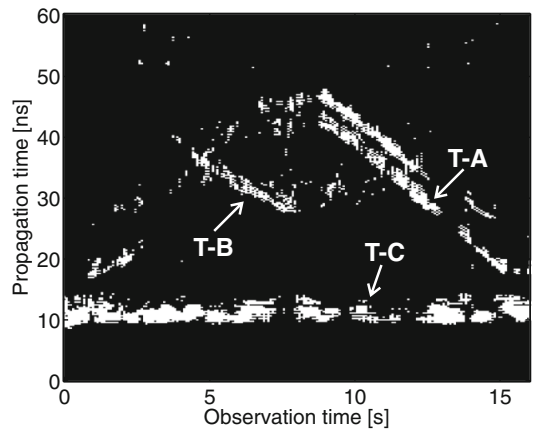

(c)

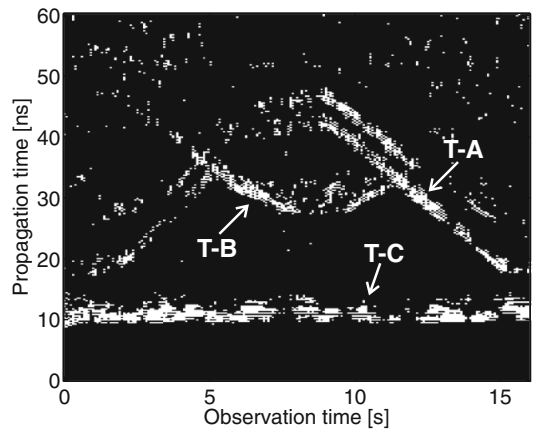

(d)

Fig. 9 Illustration of the impact of weak signal enhancement application and radar antenna location on target detection. Scenario 2 , sensor S1. The detector outputs for the receiving channel $R x_{1,1}: \mathbf{a}$ antenna altitude $1.35 \mathrm{~m}$, no weak signal enhancement has been used; b antenna altitude $1.35 \mathrm{~m}$, advanced normalization has been used; $\mathbf{c}$ antenna altitude $1.85 \mathrm{~m}$, no weak signal enhancement has been used; d antenna altitude $1.85 \mathrm{~m}$, advanced normalization has been used 


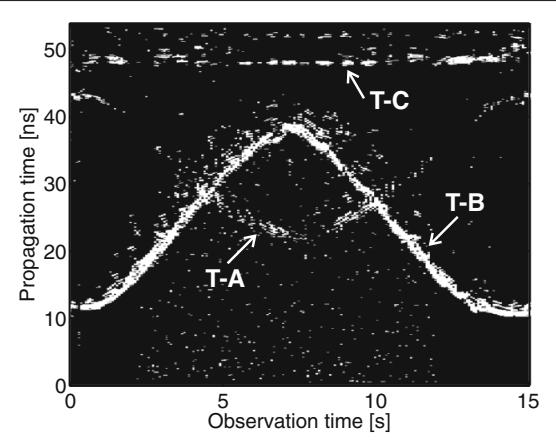

(a)

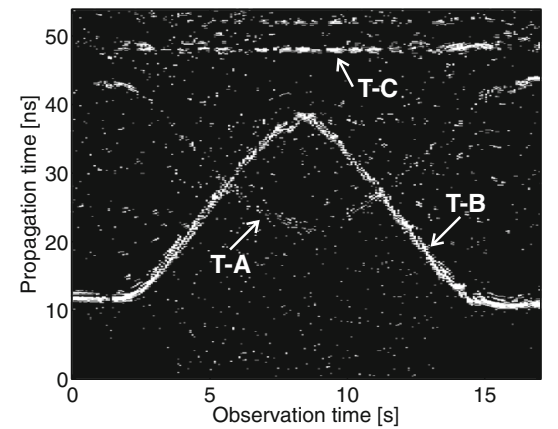

(b)

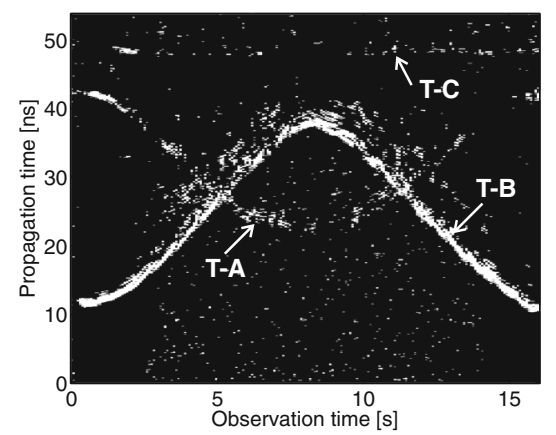

(c)

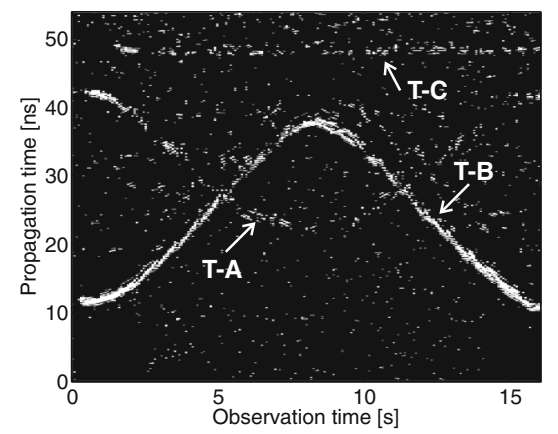

(d)

Fig. 10 Illustration of the impact of weak signal enhancement application and radar antenna location on target detection. Scenario 2, sensor S2. The detector outputs for the receiving channel $R x_{2,1}: \mathbf{a}$ antenna altitude $1.35 \mathrm{~m}$, no weak signal enhancement has been used; b antenna altitude $1.35 \mathrm{~m}$, advanced normalization has been used; $\mathbf{c}$ antenna altitude $1.85 \mathrm{~m}$, no weak signal enhancement has been used; d antenna altitude $1.85 \mathrm{~m}$, advanced normalization has been used
- The antenna altitude has been set to $1.35 \mathrm{~m}$ (hereinafter referred to as "basic" altitude) or to 1.85 m ("higher" altitude).

Let us now analyze the results achieved by the sensor $\mathrm{S} 1$ for the scenario 2. As can be observed in Fig. 9, each of the proposed approaches has provided a small improvement in such a challenging scenario. Unfortunately, none of them has removed the consequences of the mutual shielding completely. The static shielding of the target T-A has been the most suppressed by the weak signal enhancement. However, its utilization has resulted in a higher amount of false alarms (Fig. 9b, d). The higher setting of the sensor antennas has strengthened the T-A primary trace in its farther parts but almost totally depressed it in the first part of the measurement (Fig. 9c, d). Dynamic shadowing of the target T-B has been suppressed only by the combination of both methods (Fig. 9d). However, the obtained T-B primary trace has been insufficient for a track creation. And finally, because the target T-C was located directly opposite to $T x_{1}$, it has been perfectly detected.

The comparison of all approaches considered for the sensor S2 is shown in Fig. 10. As the target T-C was situated too far from the sensor antennas, dynamic shadowing between T-A and T-B has manifested in the detector outputs. It can be seen in Fig. 10 that none of the methods has aggravated the good detection results of target T-B. Additionally, the primary trace of the shielded target T-A has been subtly enhanced by every method. More expressive improvement of the T-A detection has been obtained only for short instants by the higher setting of the antennas (Fig. 10c, d). The T-C was dynamically shadowed by the T-A and T-B. The worse results for the target T-C was caused by the higher setting of the sensor antennas. On the other hand, the application of advanced normalization has provided some improvement of the detection of T-C in comparison with the approach where no signal enhancement has been applied.

The detection outputs provide the first important results within tracking scenarios. However, the final results depend in a substantial way on the results of the subsequent signal processing phases such as TOA estimation, trace connection, localization and MTT. Their impact is illustrated in Section 5, where all approaches that reduce the impact of the mutual shielding effect are considered.

\section{UWB sensor network application}

The alternative approach on how to improve target tracking is to use two or more radars forming UWB sensor network for monitoring the same area at the same time. It has been shown in [44-46] that this concept possesses the great potential to increase moving person detection 
probability, to improve person localization accuracy and to extend the coverage of a monitored area. Experience with multiple moving person detection has shown that the target located closest to a radar antenna array is detected with the highest probability. This observation can be explained by the basic radar equation and the fact that this target is not shadowed by the remaining targets. Following this consideration, an optimal number of sensor nodes should match the number of the expected targets, and simultaneously, the node arrangement should be symmetrically spread around the monitored area. For example, if four UWB sensors are deployed in the four corners inside the room for the scenarios analyzed in this paper (Figs. 1 and 2), the originally shielded parts of the monitored area will be illuminated, and hence, the persons located inside the room will be detected and localized. However, if the total number of the UWB sensors is restricted (e.g. due to their cost or availability) or if locations of the sensors are limited/constrained (e.g. for through-the-wall scenarios considered in this paper), then the number of the sensors will not match the number of the targets, and hence, a smaller number of sensors can be deployed only. In this case, the approaches proposed in our paper could be helpful and reasonable.

The UWB sensor network dedicated to short-range detection and tracking of multiple persons (e.g. [39]) consists of a set of basic nodes and a central node (a data fusion center) connected by a WLAN. As the basic nodes, the single UWB radars equipped with one transmitting and two receiving antennas and an onboard signal processor unit are assumed. This concept of the UWB radar network allows modification of the network node number in a flexible way. The main advantage of the sensor network created in such a way is the possibility of using the same signal processing procedure for tracking of multiple moving targets as that described in Section 2 (Fig. 7, Fig. 8). The raw signals acquired by every node of the UWB sensor network are independently processed up to the localization phase. Then, the coordinates of the detected targets estimated by the particular nodes are provided to the fusion center (central node). There the target coordinates are time-synchronized and transformed into the common coordinate system. On the basis of data fused in this manner, the final target tracks are estimated by the same MTT system as in the case of the single sensor application (Section 2). More details about the concept of the UWB sensor network as well as achieved experimental results can be found in $[31,39,42]$.

The inputs and the outputs of the MTT implemented within the data fusion center in both considered scenarios are depicted in Fig. 11. The first and second columns of Fig. 11 represent time synchronized and spatially transformed target coordinates estimated by the nodes $\mathrm{S} 1$ and S2, respectively. The final tracks obtained by two-node
UWB sensor network are given in the last column. It can be seen in Fig. 11c that the estimated tracks in scenario 1 correspond very well to the true trajectories of the targets (Fig. 1). UWB sensor network with two nodes located against each other correctly detected and tracked two mutually shielding persons.

The tracks estimated for scenario 2 are depicted in Fig. 11f. They also provide correct but incomplete information about the presence of persons in the scanned classroom. From three persons, only two persons (T-B, $\mathrm{T}-\mathrm{C})$ closest to the nodes were detected and tracked in conformity with the true scenario (Fig. 2). Though the fused coordinates in Fig. 11f partly indicate the trajectory of target T-A, they do not fulfill hard conditions to start a new track. Instead of this, the presence of the blackboard as a strong and large-size reflector is found in the monitored area (magenta circular arc in Fig. 11f). The explanation of this effect referred to as a defective localization of the target is beyond the scope of this paper and will not be analyzed here. Its description can be found in [59]. And finally, the estimated positions of the T-C are depicted by the red spots located in front of sensor S2 (Fig. 11f).

As we have previously stated, the optimal number of the sensor network nodes should at least match the number of the expected targets, while the node arrangement should be symmetrically spread around the monitored area. Then, it can be assumed that the third node of the sensor network located behind the wall at the right side of the monitored region would with a high probability be able to detect and track also the remaining target at scenario 2 (target T-A). However, a sufficient number of radar nodes cannot be always used because of their absence or because of missing access to the desired position. In such cases, some subsidiary approaches are required for suppressing the mutual shielding effect. The methods discussed in the previous section can be used with advantage for the solution of that problem.

Two challenging scenarios were chosen in this paper for the demonstration of dynamic and static shielding between targets tracked by an UWB sensors. The complexity of the scenarios has resulted from the fact that in a relatively small, fully furnished room, several persons were tracked through thick concrete walls. Moreover, the sensor antennas could be placed only in a limited space due to the presence of strong reflectors (Fig. 3a, blackboards covered by a thin layer of colour with non-zero conductance). In the previous paragraphs of this section, we have shown that two-node UWB sensor network with centralized architecture completely solves the mutual shielding problem in scenario 1 represented by two moving persons.

However, the same network has been capable of tracking only two persons from the three present in scenario 2 . With the aim of solving this task with the two-node UWB 


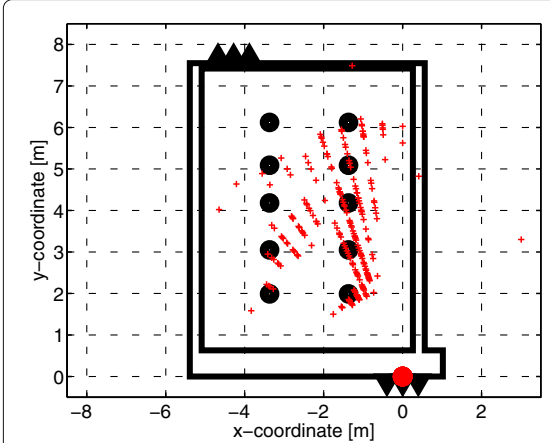

(a)

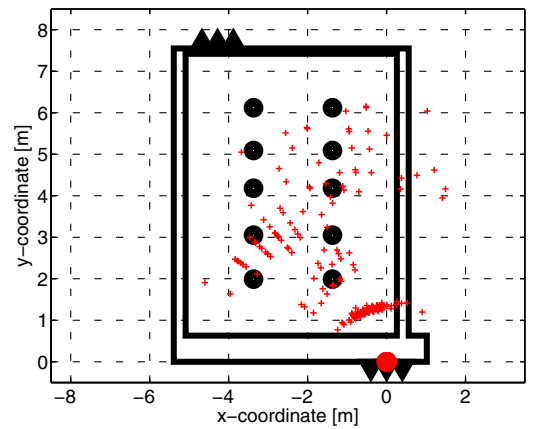

(d)

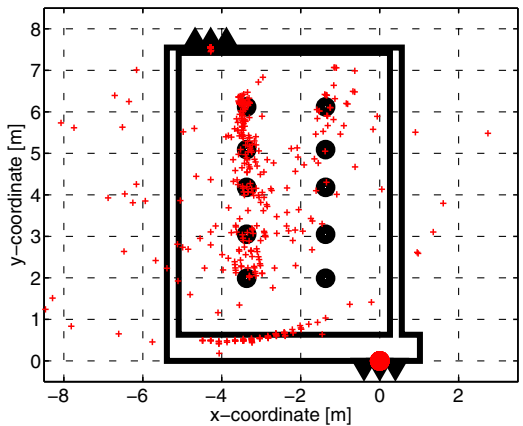

(b)

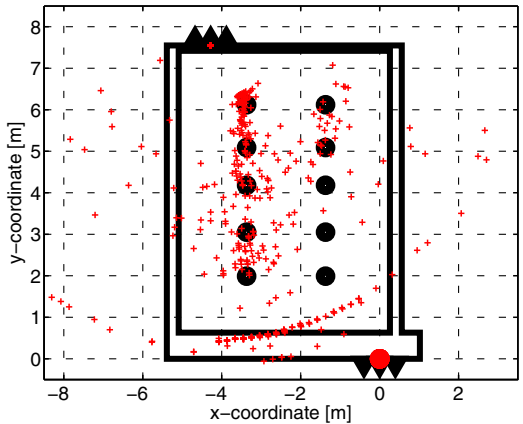

(e)

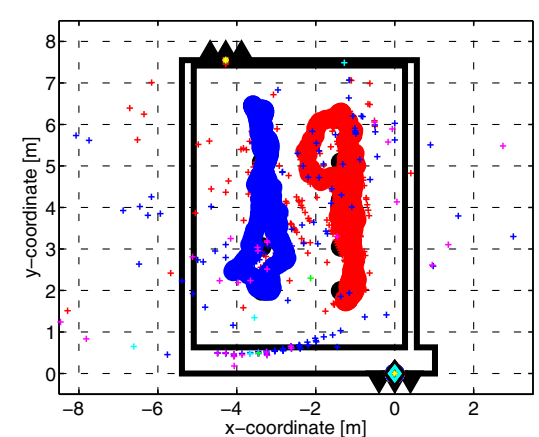

(c)

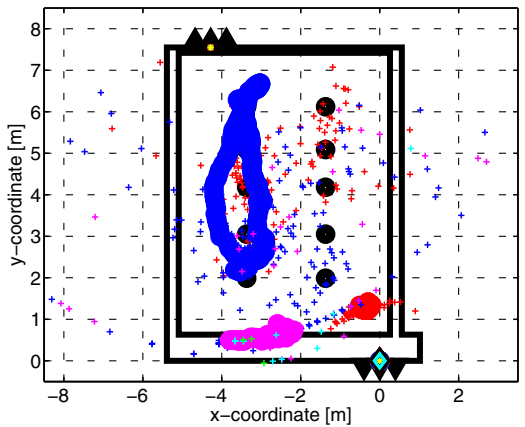

(f)

Fig. 11 Target localization and tracking by the UWB sensor network. Scenario 1: a input data of MTT from sensor S1, b input data of MTT from the sensor S2, c the fusion center output - the final target track estimate. Scenario 2: $\mathbf{d}$ input data of MTT from sensor S1, e input data of MTT from sensor S2, $\mathbf{f}$ the fusion center output - the final target track estimate

sensor network, two additional approaches to shielding impact suppression (weak signal enhancement, optimized location of antenna array) and their combination have been applied. The obtained results are summarized in Fig. 12. In this figure, the outputs of the localization and tracking phase for scenario 2 from autonomously working sensor S1 (the first column of Fig. 12), autonomously working sensor S2 (the second column of Fig. 12) and finally from the UWB sensor network consisting of nodes S1 and S2 (the third column of Fig. 12) are given. On the other hand, the first row of Fig. 12 represents the results obtained by the standard signal processing procedure (i.e. no weak signal enhancement is applied) where the sensor antennas were located at the standard height of $1.35 \mathrm{~m}$. The next rows of Fig. 12 gradually present the results obtained by the weak signal enhancement method of raw data measured by the sensor antennas located at the relative altitude of $1.35 \mathrm{~m}$, the results obtained by the standard UWB signal processing where the sensor antennas were located at the relative altitude of $1.85 \mathrm{~m}$ and, finally, the results obtained by the weak signal enhancement method of raw data measured by the sensor antennas located at the relative altitude of $1.85 \mathrm{~m}$.

The analyses of the results given in Fig. 12 have shown that the significant improvement of the tracking results in scenario 2 was reached only by the application of the UWB sensor network in conjunction with additional approaches of shielding effect suppression (Fig. 12f, i, l). It is noteworthy that the final target tracks are not only a simple union of the tracks obtained by nodes S1 and $\mathrm{S} 2$. The real contribution of the network fusion center is its capability of creating new and/or more accurate tracks using also the isolated target locations obtained from all nodes. From the tracking results depicted in Fig. 12f, i, l, the combination of the optimized setting of the sensor antennas and weak signal enhancement provided the best conformity with the true target trajectories in scenario 2 (Fig. 12l). The self-employed optimized setting of the antenna array (Fig. 12i) also achieved very promising results.

\section{Conclusions}

In this paper, we have dealt with through-the-wall detection, localization and tracking of multiple humans by a single UWB sensor and UWB sensor network as well. We have presented that even just two persons moving in a relatively small area (scenario 1 ) can create a space and time variable shadow region with non-homogeneous spacing of additional attenuation. The subsequent scenario (scenario2) has showed that with the increasing number of the 


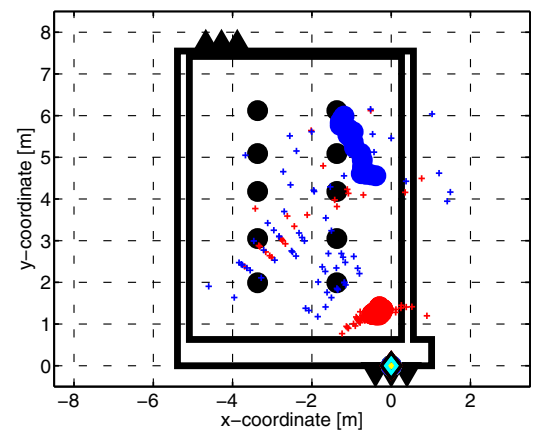

(a)

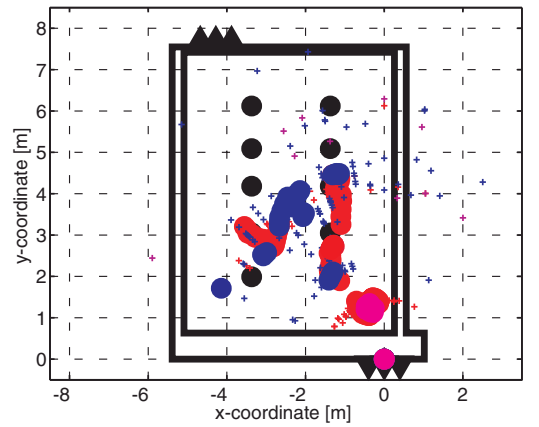

(d)

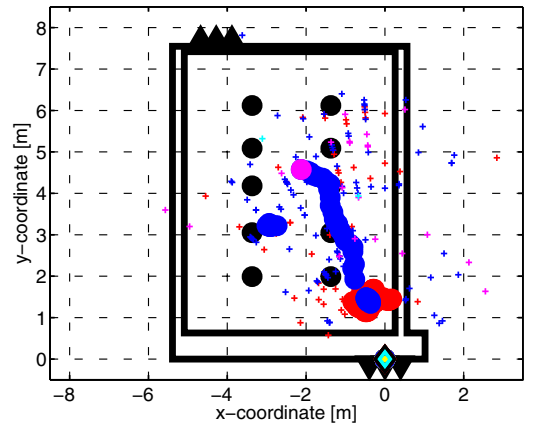

(g)

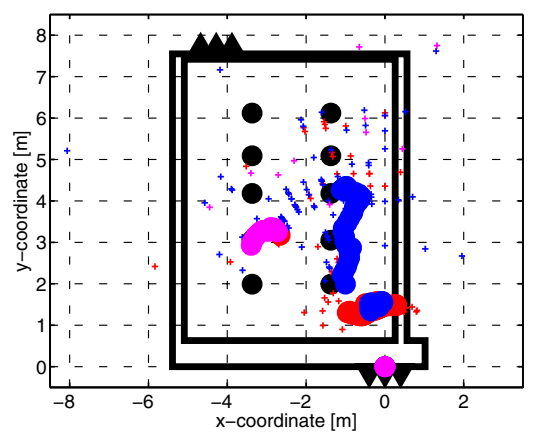

(j)

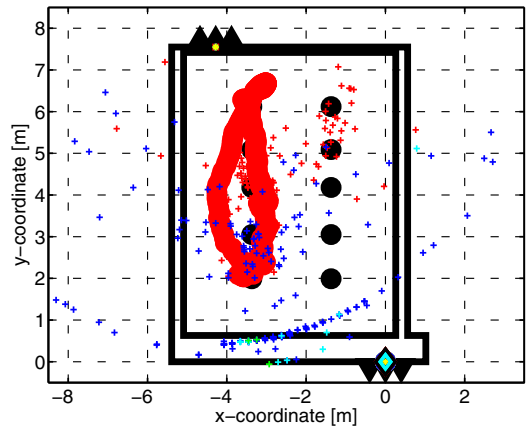

(b)

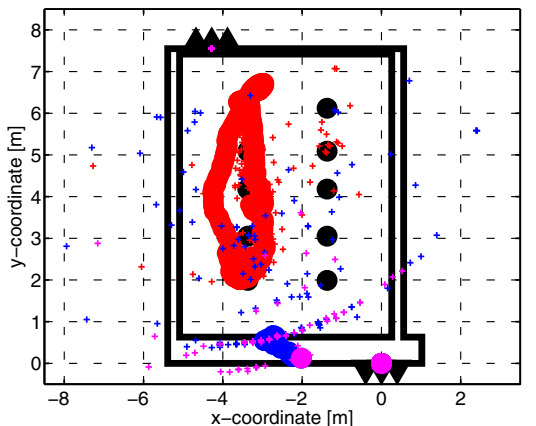

(e)

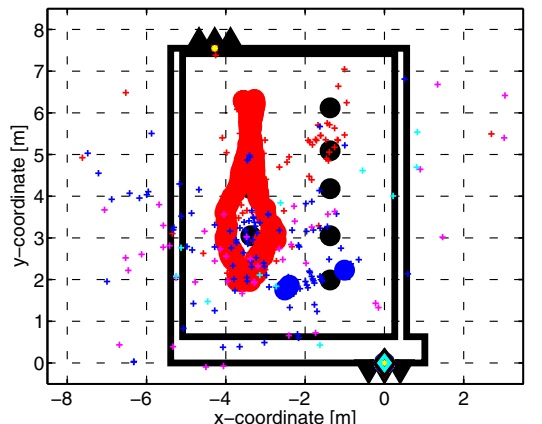

(h)

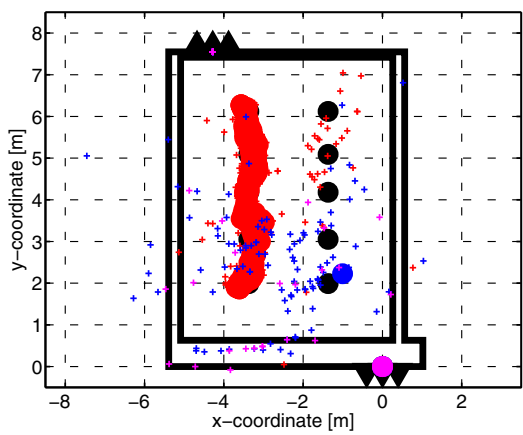

(k)

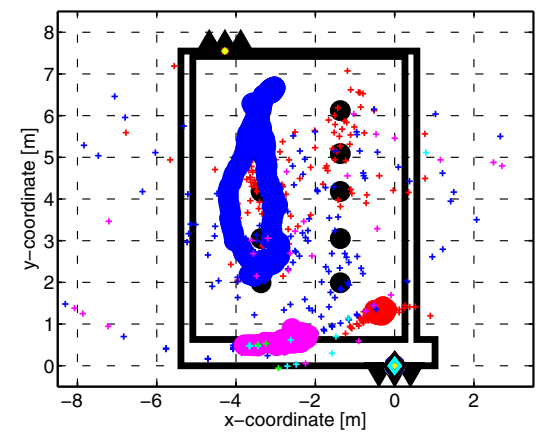

(c)

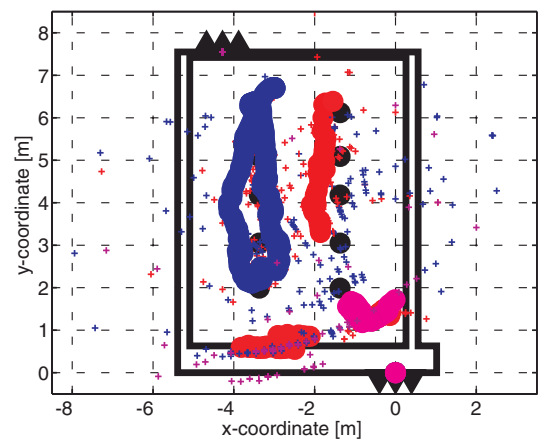

(f)

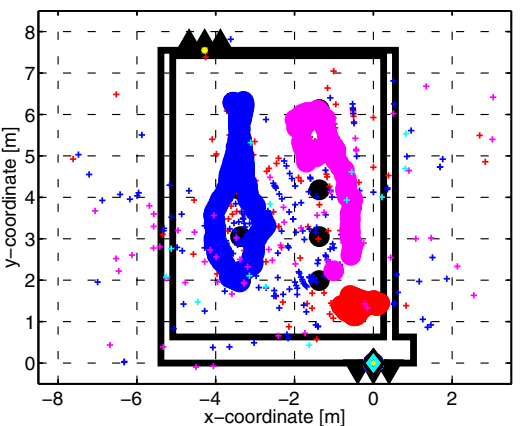

(i)

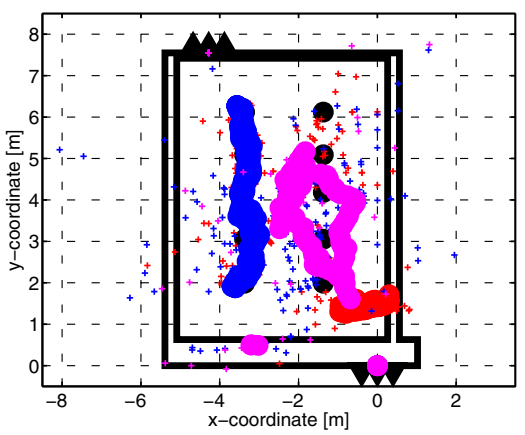

(I)

Fig. 12 Tracking results for scenario 2. Standard location of antennas and standard signal processing: a autonomously working sensor S1, b autonomously working sensor $\$ 2$ and c two-node sensor network. Standard location of antennas and weak signal enhancement: d autonomously working sensor S1, e autonomously working sensor S2 and $\mathbf{f}$ two-node sensor network. Optimized location of the antennas \& standard signal processing: $\mathbf{g}$ autonomously working sensor S1, h autonomously working sensor S2, i two-node sensor network. Optimized location of the antennas and weak signal enhancement: $\mathbf{j}$ autonomously working sensor S1, k autonomously working sensor S2 and I two-node sensor network 
tracked persons and/or with their proximity to the sensor antenna system, the detection of all targets located in the monitored area becomes very challenging.

In order to solve the outlined problem, several complementary approaches having the potential to improve multiple moving target tracking in comparison with that of standard methods have been discussed. These approaches include the enhancement of the low-level echo of the targets, the radar antennas positioned at a convenient height, application of the UWB sensor network and, finally, their mutual combinations. As has been shown, each approach can provide some improvement of multiple target tracking. Weak echo enhancement and the proper location of the radar antenna array can be employed also by a single UWB sensor. However, the importance of the mentioned approaches lies particularly in their complementarity. That is, each approach contributes to the solution of the problem of multiple targets tracking in a different way. The enhancement of the low-level echo of the targets results in the increase of echo-to-clutter and noise ratio and hence better input for the target detectors. On the other hand, the radar antenna array location at the height or slightly above height of the target heads reduces the additional attenuation level due to shielding effect. Finally, the improvement provided by the UWB sensor network application is based on the diversity principle employed for monitoring of the area of interest by a set of properly located sensors. Moreover, these approaches are also implemented in the various successive phases of the mentioned radar signal processing procedure. As we can see, the total improvement of target tracking provided by the combination of all the discussed methods is greater than that of a simple union of the contributions of the particular methods. This statement has been confirmed by the results obtained for scenario 1 and scenario 2 . Therefore, it can be concluded that the combination of the enhancement of the low-level echo of the targets, the optimized location of the radar antennas and UWB sensor network application represents a powerful tool for multiple moving persons tracking in a complex environment.

Notwithstanding the good results in the field of multiple persons tracking presented in this paper, we can see still space for their further improvement and coming closer to real-life conditions and applications. The ideas that are attractive from this point of view include the application of the extended method of the background subtraction allowing to solve the problem of the defective localization of targets [59], a solution of the problem of the detection of persons locating in close proximity and an optimization of the layout of the antennas of the sensor network nodes throughout the monitored area. The combinations of these ideas with the approaches introduced in this article are the challenging topics for our follow-up research.

\section{Acknowledgements}

This work was supported by the Slovak Research and Development Agency under the contract No. APW-0404-12.

\section{Competing interests}

The authors declare that they have no competing interests.

Received: 12 September 2016 Accepted: 27 February 2017

Published online: 17 April 2017

\section{References}

1. E Zaikov, in 11th International, Radar Symposium (IRS). UWB radar for detection and localization of trapped people (IEEE, Vilnius, 2010). doi:10.5772/10004

2. B Yamauchi, Fusing ultra-wideband radar and LIDAR for small UGV navigation in all-weather conditions. Int. Soc. Opt. Eng. (SPIE). 7692, 1-10 (2010)

3. R Salman, I Willms, A mobile security robot equipped with UWB-radar for super-resolution indoor positioning and localisation applications. Int. Conf. Indoor Positioning Indoor Nav (IPIN), 1-8 (2012)

4. J Sachs, Handbook of Ultra-Wideband Short-Range Sensing. (Wiley-VCH, Weinheim, 2013)

5. A Nezirovic, AG Yarovoy, LP Ligthart, Signal processing for improved detection of trapped victims using UWB radar. IEEE Trans. Geosci. Remote Sens. 48, 2005-2014 (2009)

6. M Survey, in Sensor, Surveillance, and Biometric Technologies (SSBT), Center of Excellence (COE). Through-the-wall sensors for law enforcement (Sensor, Surveillance, and Biometric Technologies Center of Excellence, 2012), pp. 1-31. https://www.nij.gov/topics/technology/detection-surveillance/ enhanced-surveillance/pages/through-wall.aspx. Accessed Oct 2012

7. D Porcino, J Sachs, R Zetik, in UWB Communication Systems a comprehensive Overview, ed. by MG d Benedetto, T Kaiser, AF Molish, I Oppermann, C Politano, and D Porcino. UWB ranging (Hindawi Publishing Corporation, New York, 2006), pp. 411-446

8. J Sachs, et al, in 5th European Radar Conference (EuRAD 2008). Detection and tracking of moving or trapped people hidden by obstacles using ultra-wideband pseudo-noise radar, (Amsterdam, 2008), pp. 408-411

9. C Kocks, E Scheiber, D Xu, A Viessmann, S Wang, in 2nd International Symposium on Applied Sciences in Biomedical and Communication Technologies (ISABEL 2009). A localization and tracking application for UWB (IEEE, 2009), pp. 1-5. doi:10.1109/ISABEL.2009.5373696

10. WZ Li, Z Li, H Lv, GH Lu, Y Zhang, X Jing, S Li, JQ Wang, A new method for non-line-of-sight vital sign monitoring based on developed adaptive line enhancer using low centre frequency uwb radar. Prog. Electromagn. Res. 133, 535-554 (2013)

11. B Levitas, I Naidionova, J Matuzas, in Radar Symposium (IRS), 2014 15th International. UWB radar for breath and movement detection (IEEE, 2014), pp. 1-4. doi:10.1109/IRS.2014.6869188

12. R Zetik, RS Thoma, in Ultra-WideBand (ICUWB), 2014 IEEE International Conference on. Middle-range surveillance by UWB radar: an experimental feasibility study (IEEE, 2014), pp. 129-134. doi:10.1109/ICUWB.2014.6958964

13. J Yan, H Zhao, Y Li, L Sun, H Hong, X Zhu, in 2016 IEEE Topical Conference on Biomedical Wireless Technologies, Networks, and Sensing Systems (BioWirelesS). Through-the-wall human respiration detection using impulse ultra-wide-band radar (IEEE, 2016), pp. 94-96. doi:10.1109/BIOWIRELESS.2016.7445572

14. C Kim, J-Y Lee, ToA-based multi-target localization and respiration detection using UWB radars. EURASIP J. Wirel. Commun. Netw. 2014(1), $1-15$ (2014)

15. J Sachs, M Helbig, R Herrmann, M Kmec, K Schilling, E Zaikov, Remote vital sign detection for rescue, security, and medical care by ultra-wideband pseudo-noise radar. Ad Hoc Netw. 13, Part A, $42-53$ (2014). http://dx.doi. org/10.1016/j.adhoc.2012.07.002

16. E Zaikov, J Sachs, in Ultra Wideband, ed. by B Lembrikov. UWB radar for detection and localization of trapped people (InTech, Croatia, 2010), pp. 323-346

17. J Sachs, M Helbig, R Herrmann, M Kmec, E Zaikov, P Rauschenbach, in 1st International Conference on Wireless Technologies for Humanitarian Relief. Trapped victim detection by pseudo-noise radar (ACM, New York, 2011), pp. 265-272. doi:10.1145/2185216.2185289 
18. J Sachs, M Helbig, R Herrmann, M Kmec, K Schilling, E Zaikov, Remote vital sign detection for rescue, security, and medical care by ultra-wideband pseudo-noise radar. Ad Hoc Netw. 13(Part A), 42-53 (2014)

19. J Sachs, R Herrmann, M-sequence-based ultra-wideband sensor network for vitality monitoring of elders at home. IET Radar Sonar Navig. 9(2), 125-137 (2015)

20. P Withington, H Fluhler, S Nag, Enhancing homeland security with advanced UWB sensors. IEEE Microw. Mag. 4(3), 51-58 (2003)

21. S Chang, M Wolf, JW Burdick, Human detection and tracking via ultra-wideband (UWB) radar. IEEE Int. Conf. Robot. Autom, 453-457 (2010)

22. F Folster, $\mathrm{H}$ Rohling, Data association and tracking for automotive radar networks. IEEE Trans. Intell. Transport. Syst. 6(4), 370-377 (2005)

23. J Rovňaková, Complete signal processing for through wall tracking of moving targets. (Lambert Academic Publishing, Germany, 2010)

24. V-H Nguyen, J-Y Pyun, Location detection and tracking of moving targets by a 2 d ir-uwb radar system. Sensors. 15(3), 6740 (2015). http://www. mdpi.com/1424-8220/15/3/6740

25. J Li, L Liu, Z Zeng, F Liu, in 14th International Conference on Ground Penetrating Radar (GPR). Simulation and signal processing of UWB radar for human detection in complex environment (IEEE, 2012), pp. 209-213. doi:10.1109/ICGPR.2012.6254862

26. J Rovňaková, D Kocur, in EURASIP Journal on Wireless Comm. and Networking, The special issue: Radar and Sonar Sensor Networks. TOA estimation and data association for through wall tracking of moving targets. Vol. 2010 (Springer International Publishing, 2010). doi:10.1155/2010/420767

27. SS Blackman, R Popoli, Design and analysis of modern tracking systems. (Artech House Publishers, Norwood, 1993)

28. D Kocur, J Rovňaková, D Urdzík, in IEEE 7th Int.Symposium on Intelligent Signal Processing (WISP 2011). Experimental analyses of mutual shadowing effect for multiple target tracking by UWB radar (IEEE, 2011), pp. 91-94. doi:10.1109/WISP.2011.6051690

29. D Kocur Rovňaková, D Urdzík, Short-range UWB radar application: problem of mutual shadowing between targets. Elektrorevue. 2(4), 37-43 (2011). ISSN 1213-1539

30. D Urdzík, et al, in Progress In Electromagnetics Research Symposium (PIERS 2012). Shadowing effect investigation for the purposes of person detection and tracking by UWB radars (The Electromagnetics Academy, Cambridge, 2012), pp. 834-837. https://piers.org/piersproceedings/ download.php?file=cGllenMyMDEyS3VhbGFMdW1wdXJ8MIA1XzA4NjQ ucGRmfDExMDkyMDA5MjQXNg==

31. J Fortes, D Kocur, in Int. IEEE Conf. on Microwaves, Communications, Antennas and Electronic Systems (COMCAS 2013). Solutions of mutual shadowing effect between people tracked by UWB radar (IEEE, 2013). doi:10.1109/COMCAS.2013.6685300

32. SH Chang, et al, People tracking with UWB radar using a multiple-hypothesis tracking of clusters (MHTC) method. Int. J. Soc. Robot. 2(1), 3-18 (2010)

33. B Zhang, W Wang, Through-wall detection of human being with compressed UWB radar data. EURASIP J. Wirel. Commun. Netw. 2013(1), 162 (2013)

34. R Zetik, et al, in Proc.of SPIE - Defense \& Security Symposium. Detection and localization of persons behind obstacles using M-sequence through-the-wall radar, Vol. 6201 (Sensors, and Command, Control, Communications, and Intelligence (C3I) Technologies for Homeland Security and Homeland Defense, 2006). doi:10.1117/12.667989

35. ZW Mekonnen, A Wittneben, in Dresden, Germany: 8th Workshop on Positioning, Navigation and Communication (WPNC). Localization via Taylor series approximation for UWB based human motion tracking (IEEE, 2011). doi:10.1109/WPNC.2011.5961019

36. MG Amin, F Ahmad, Change detection analysis of humans moving behind walls. IEEE Trans. Aerosp. Electron. Syst. 49(3), 1410-1425 (2013) doi:10.1109/TAES.2013.6557995

37. G Shingu, K Takizawa, T Ikegami, in Parallel and Distributed Computing, Applications and Technologies (PDCAT 2008) Int.Conf. on. Human body detection using MIMO-UWB radar sensor network in an indoor environment (IEEE, 2008), pp. 437-442. doi:10.1109/PDCAT.2008.66

38. R Zetik, S Jovanoska, R Thoma, in IEEE Int.Conf. on Ultra Wideband (ICUWB). Simple method for localisation of multiple tag-free targets using UWB sensor network (IEEE, 2011), pp. 268-272. doi:10.1109/ICUWB.2011.6058843
39. J Rovňaková, D Kocur, in The 8th European Radar Conference (EuRAD 2011). Short range tracking of moving persons by UWB sensor network (IEEE, 2011), pp. 321-324. http://ieeexplore.ieee.org/document/6101097/

40. D Urdzík, R Zetik, D Kocur, J Rovňáková, in Proc. of the German Microwave Conference (GeMiC 2012). Shadowing effect analysis at multiple moving persons tracking by UWB radar (IEEE, 2012), pp. 1-4. http://ieeexplore. ieee.org/document/6185212/

41. J Rovňaková, D Kocur, Weak signal enhancement in radar signal processing. Radioelektronika, 147-150 (2010)

42. J Rovňáková, D Kocur, in 21st International Conference Radioelektronika Data fusion from (UWB) radar network: preliminary experimental results (IEEE, 2011). doi:10.1109/RADIOELEK.2011.5936439

43. E Fishler, A Haimovich, R Blum, L Cimini, D Chizhik, R Valenzuela, Spatial diversity in radars-models and detection performance. IEEE Trans. Signal Process. 54(3), 823-838 (2006)

44. S Bartoletti, A Giorgetti, A Conti, in Proc.of Tyrrhenian Workshop on Advances in Radar and Remote Sensing. UWB sensor radar networks for indoor passive navigation (IEEE, 2012), pp. 140-145. doi:10.1109/TyWRRS.2012.6381119

45. R Thoma, O Hirsch, J Sachs, R Zetik, in The Second European Conference on Antennas and Propagation, 2007. EUCAP 2007. UWB sensor networks for position location and imaging of objects and environments (IET, 2007), pp. 1-9. doi:10.1049/ic.2007.1336

46. M Chiani, A Giorgetti, M Mazzotti, R Minutolo, E Paolini, in Proc.of the IEEE International Conference on Ultra-Wideband (ICUWB). Target detection metrics and tracking for UWB radar sensor networks (IEEE, 2009), pp. 469-474. doi:10.1109/ICUWB.2009.5288758

47. Farlex, McGraw-Hill Dictionary of Scientific \& Technical Terms. (The McGraw-Hill Companies, Inc., 2003). http://encyclopedia2. thefreedictionary.com/radar+shadow

48. K Watanabe, S Hari, K Ohno, T Ikegami, in International Symposium on Communications and Information Technologies (ISCIT 2008). Experiments on shadow effects of body and effective paths for UWB transmission in ban (IEEE, 2008), pp. 232-237. doi:10.1109/ISCIT.2008.4700189

49. D Andreuccetti, R Fossi, C Petrucci, Calculation of the dielectric properties of human body tissues in the frequency range $10 \mathrm{~Hz}-100 \mathrm{GHz}$. (Italian National Research Council, Institute for Applied Physics, Florence, Italy. Available: http://niremf.ifac.cnr.it/tissprop/. Accessed 10 June 2016

50. D Urdzík, Contribution to the analysis of multiple moving target tracking by UWB radar (2012). Ph.D. dissertation, Technical University of Košice, Letná 9, 04120 Košice, Slovakia

51. Antenna specification, RFspin s.r.o. (2003). http://www.rfspin.cz/en/ antennas/drh10.php. Accessed 10 June 2016

52. D Kocur, J Rovňaková, in Microwave and Milimeter Wave Circuits and Systems - Emerging Design, Technologies and Applications, ed. by A Georgiadis, H Rogier, L Roselli, and P Arcioni. Short-range tracking of moving targets by handheld UWB radar system. (Wiley, 2012)

53. G Minkler, J Minkler, CFAR: The Principles of Automatic Radar Detection in Clutter. Magellan Book Company, 1990)

54. PK Dutta, AK Arora, SB Bibyk, in Proc.of the Fifth International Conference on Information Processing in Sensor Networks. Towards radar-enabled sensor networks (IEEE, 2006), pp. 467-474. doi:10.1145/1127777.1127848

55. J Rovňáková, D Kocur, Compensation of wall effect for through wall tracking of moving targets. Radioengineering. 18(2), 189-195 (2009)

56. D Kocur, M Švecová, J Rovňáková, Through-the-wall localization of a moving target by two independent ultra-wideband (UWB) radar systems. Sensors. 13, 11969-11997 (2013). doi:10.3390/s130911969. http://www. mdpi.com/1424-8220/13/9/11969

57. D Kocur, J Rovňaková, M Švecová, in Towards Intelligent Engineering and Information Technology, ed. by IJ Rudas, J Fodor, and J Kacprzyk. Through wall tracking of moving targets by M-sequence UWB radar (Springer, Springer-Verlag Berlin Heidelberg, 2009)

58. W Xingxing, J Zhong-ping, D Repperger, Y Guo, in Mechatronics and Automation, Proc.of the IEEE International Conference. Nonlinear enhancement of weak signals using optimization theory (IEEE, 2006). doi:10.1109/ICMA.2006.257454

59. D Kocur, D Novák, J Rovňáková, in IEEE 9th Int.Symposium on Intelligent Signal Processing (WISP 2013). Moving person tracking by UWB radar system in complex environment (IEEE, 2013). doi:10.1109/WISP.2013.6657486 